Original Research

\title{
Contactless Detection of Natural Bending Frequencies using Embedded Metallic-Glass Ribbons inside Plastic Beams made of 3-D Printing
}

\author{
Dimitris Kouzoudis *, Georgios Samourgkanidis, Christos I. Tapeinos
}

Department of Chemical Engineering, University of Patras, 26504, Rio, Greece; E-Mails: kouzoudi@upatras.gr; g.samourgkanidis@gmail.com; tapeinoschristos@gmail.com

* Correspondence: Dimitris Kouzoudis; E-Mail: kouzoudi@upatras.gr

Academic Editor: Waseem Haider

Special Issue: Recent Advances in Metallic Glass

Recent Progress in Materials

2021, volume 3 , issue 2

doi:10.21926/rpm.2102010
Received: December 12, 2020

Accepted: March 15, 2021

Published: April 06, 2021

\begin{abstract}
In the current work, to identify the bending mode harmonics, 30 microns thin magnetoelastic ribbons made of metallic glass are embedded inside $6 \mathrm{~mm}$ thick PLA plastic cantilever beams made by 3-D printing. This is possible because the ribbons are of magnetoelastic nature and thus change their mechanical state inducing a corresponding change in their magnetic state. The ribbons are placed at four different depths, starting with zero depth at the beam's external surface all the way inside to the beam's mid-plane. This technique is capable of detecting seven harmonics, and remarkably, these frequencies remain the same within a marginal error of $1 \%$ for all the depths. The amplitude of the modes drops with the increase in depth but is still strong enough, except at the midplane, to be used as a sensing signal. The harmonics spectrum is the unique signature of the structure's state; this is a proof of concept that in a contactless fashion, the embedded ribbons provide useful information about the mechanical health of a structure.
\end{abstract}

\section{Keywords}

Magnetoelastic; metallic glass sensors; bending frequencies

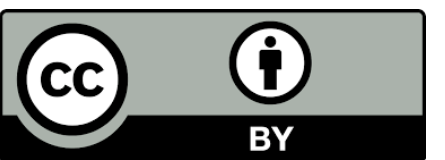

(C) 2021 by the author. This is an open access article distributed under the conditions of the Creative Commons by Attribution License, which permits unrestricted use, distribution, and reproduction in any medium or format, provided the original work is correctly cited. 


\section{Introduction}

Mechanical structures are subject to adverse conditions during their use, which affects their applications. Most damage is caused by the formation and spread of cracks in the materials. Therefore, the detection and characterization of such faults prevent the material from failing, thereby ensuring its smooth operation. Two crucial areas of research that deal with the above are Non-Destructive Evaluation (NDEs) and Structural Health Monitoring (SHM). The most commonly used techniques are based on mechanical voltage sensors (electric or fiber optics [1]), piezoelectric and acoustic sensors (acoustic emission and wave propagation control [2]), and accelerometers. New approaches include Micro Electrochemical Systems (MEMS) [3, 4], piezoelectric paints, Large Area Electronics (LAE), and contactless laser techniques. Nonetheless, there are several algorithmic data analysis methods combined with sensor techniques for the detection and characterization of cracks [5].

Some earlier studies [6-8] explored the use of a continuous grid of piezoelectric lead-zirconatetitanate (PZT) (PZT) elements, which are recording vibrations and mechanical stresses toward the crack detection in mechanical structures. With the help of wavelet transform, Okafor and Dutta [9] recorded and analyzed the first six mode shapes of a damaged and undamaged aluminum cantilever beam using a scanning laser vibrometer. Jassim et al. [10] presented a nice mini-review on vibration analysis for a damage occurrence of a cantilever beam. Structural fault recognition based on vibration theory has offered promising results in engineering studies [11, 12]. The work presented earlier [13] examined the detection of cracks according to changes in curvature mode shapes. A cantilever and an analytical model were used to explain that the change in the curvature of the mode shapes is located in the area of the crack. Adams et al. [14] used the reduction in harmonics and the increase in damping to detect cracks in fiber-reinforced plastics. Loland et al. [15] and Vandiver [16] used the same principles to identify damage in coastal structures. From the relative change in physical frequencies, they were able to predict the location of the fault. Yuen [17] used finite element analysis to a beam to prove that there is a relation between the change of the beam eigenvectors and the location of the damage.

Dimarogonas [18], Hondros [19], and Dimarogonas and Hondros [20, 21] modeled the crack as local flexibility calculated by a fracture in a mechanical method. They measured experimentally and developed a spectral method to identify the crack in various structures by relating the crack depth to the change in harmonics. Cawley and Adams [22] developed an experimental method to determine the location and depth of the crack based on the change in harmonics. Anifantis et al. [23] further developed the spectral method to discover the earthquake-induced faults in reinforced concrete. The fault was determined through changes in the physical frequencies of the spectrum. Inagaki et al. [24] estimated the size and location of cracks from the analysis of harmonics by static deflection in the case of transverse oscillation in rotors.

Following a similar route, our group has been a pioneer in the usage of magneto-elastic ribbons for identifying and localizing the cracks in metal cantilevers $[25,26]$ by recording the shift of their harmonics under bending vibrations. Magnetoelasticity is the change in the magnetic state of these materials due to the application of mechanical stress on them [27]. It is the magnetic analog of the 
piezo crystals in electricity. The coupling of the magnetic and elastic properties of the magnetoelastic materials makes them ideal sensors for the detection of mechanical vibrations since the produced signal (EMF voltage) is proportional to the amplitude and possesses the same frequency as the vibrational displacements. Typically, these materials are made up of amorphous metallic alloys in the form of thin ribbons known as "metallic glasses". In the presented work, the alloy Metglas 2826MB3 is referred to as "Metglas" (for properties, refer to the "Materials and Methods" section below).

As shown earlier $[25,26]$, the produced signal from an aluminum cantilever with Metglas ribbons on it can be Fourier-analyzed to produce the spectrum of the bending modes of the beam when it is subject to bending excitations. This is a unique signature of the mechanical health of this structure, and its recording is of paramount importance in damage detection. In these articles, the experimental results are combined with numerical approaches (finite element method) for the study of cracks. The presented method exhibits the following advantages over other methods:

- The magneto-elastic material is of low cost ( 0.40 euros per meter).

- Its small thickness (about 30 micrometers) possesses a minimal disturbance to the structure that is attached to it (this point will be further clarified in the "Results" section below).

- It is a stable material since it is metallic.

- The produced signal is received contactless, without any wires or other connections.

- The detection technology is easy and of low cost (requires simple electromagnetic equipment at low frequencies of a few $\mathrm{kHz}$ ).

The current work is the continuation of our previous work described above. In those works, (a) plastic is used instead of aluminum cantilever beams, and (b) the sensors are placed inside the material; this is very difficult to do with metallic beams as the material absorbs the electromagnetic radiation.

\section{Theory of Bending}

The differential equation of a thin and long cantilever beam with its length along the z-axis from $z=0$ (fixed end) to $z=L$ (free end) and its thickness along the $x$-axis is as follows:

$$
E I \frac{\partial^{4} w}{\partial z^{4}}+\rho A \frac{\partial^{2} w}{\partial t^{2}}=0
$$

where $w(z, t)$ represents the vertical deflection of the beam (along $x$ ) from its equilibrium position which is assumed to be the $x z$-plane, $t$ indicates the time, $A$ is the $x y$ beam cross-section area, $I$ represents the second moment of area (an integral of the moment of inertia across $A$ ), and finally, $E$ and $\rho$ are respectively the elastic modulus and density of the beam material. The solution of the above differential equation leads to the bending oscillation frequencies $f_{n}$ with $n=1,2,3 \ldots$ which can be represented as:

$$
f_{n}=\frac{x_{n}{ }^{2}}{2 \pi L^{2}} \sqrt{\frac{E I}{\rho A}}
$$

where the $x_{n}$ are plain arithmetic values, one for each harmonic $n$, and are mentioned in Table 1 below. 
Table 1 Values $x_{n}$ appearing in Eq. 1 describing the bending mode frequencies $f_{n}$ (harmonics) of a cantilever beam.

\begin{tabular}{llllllllllll}
\hline$n$ & 1 & 2 & 3 & 4 & 5 & 6 & 7 & 8 & 9 & 10 & 11 \\
\hline$x_{n}$ & 1.87 & 4.69 & 7.85 & 11.0 & 14.1 & 17.3 & 20.4 & 23.5 & 26.6 & 29.8 & 32.9 \\
\hline
\end{tabular}

\section{Materials and Methods}

The commercial alloy Metglas $2826 \mathrm{MB} 3$ in the form of thin ribbon was purchased from Metglas ${ }^{\circledR}$ Inc. As illustrated in Figure 1a, the demonstrated sample was a plastic beam of $200 \mathrm{~mm} \times 30 \mathrm{~mm} \times$ $6 \mathrm{~mm}$ made of PLA (Polylactic acid) material in a 3-D printer. It has two Metglas ribbons having the properties as mentioned in Table 2 below, embedded in it at different depths $d$, with zero-depth corresponding to the attachment of the ribbons at the beam's top surface. Figure $1 \mathrm{~b}$ reveals that the sample was designed in two phases in the 3-D printer: first the bottom part with a height of 6$\mathrm{d}$ millimeters, with two $100 \mu \mathrm{m}$ deep grooves in it, which is the minimum vertical step of the printer, and then the top part with a thickness of $d$ millimeters. After its completion, the bottom part was left lying on the printer bed, and two $30 \mu \mathrm{m}$ thick Metglas ribbons were placed in its grooves tightly with the help of double sticky tape (the tape was first attached on one side of the ribbons and then the ribbons were attached to the PLA bottom part). Subsequently, the printing of the top part of the thickness $d$ took place, with an initial vertical offset equal to 6 - $d$ (in $\mathrm{mm}$ ) to have a beam with a total thickness of $6 \mathrm{~mm}$. The image in Figure 1c indicates the construction of the beam in the 3D printer. Prior to their placement in the beam, the two ribbons were annealed at $300{ }^{\circ} \mathrm{C}$ for $0.5 \mathrm{~h}$ to get stronger magnetoelastic properties and thus a better signal. The anneal temperature was chosen to be below the alloy's Curie temperature of $353{ }^{\circ} \mathrm{C}$ (refer to Table 2). For the Metglas attachment on the beam, the double sticky tape was selected in place of epoxy resin since (a) it worked well in the previous work $[25,26]$ and (b) it provided a uniform attachment across the Metglas ribbons without leading to local stresses due to non-uniformity that a badly spread resin can cause during the curing. Similarly, it has a uniform thickness down to the sub-millimeter range, while it would be extremely difficult to create a resin spread of such a small and uniform thickness.

Table 2 Physical and geometrical properties of Metglas 2826MB3 sensing ribbons.

\begin{tabular}{lcc}
\hline Property & Value & \\
\hline & Fe & $40-50 \%$ \\
& $\mathrm{Ni}$ & $40-50 \%$ \\
Composition (average) & Mo & $5-10 \%$ \\
& B & $1-5 \%$ \\
Length & Co $<0.3 \%$ \\
Width & $100 \mathrm{~mm}$ \\
Thickness & $1 / 4 \mathrm{in}$ \\
\hline Density & $30 \mu \mathrm{m}$ \\
\hline
\end{tabular}




\begin{tabular}{lc}
\hline Modulus of Elasticity & $100-110 \mathrm{GPa}$ \\
Saturation Magnetostriction & $12 \mathrm{ppm}$ \\
Curie Temperature & $353^{\circ} \mathrm{C}$ \\
Saturation Induction & $0.88 \mathrm{~T}$ \\
\hline
\end{tabular}
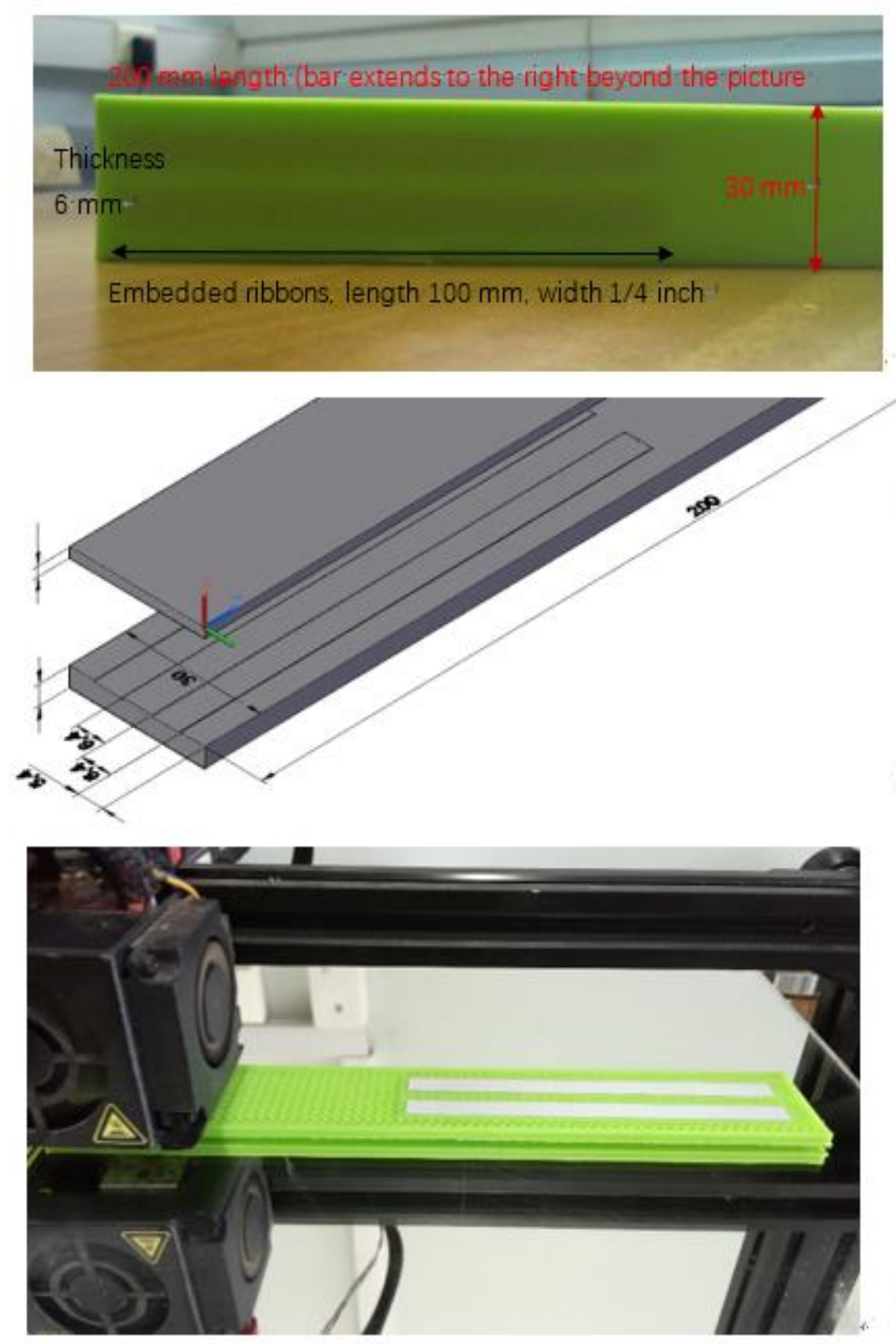

$\mathrm{C}$

Figure 1 The beam sample (a) top view photograph where the two sensing films can be visibly discerned inside the material, (b) it is designed in two parts so that it could be manufactured in the 3-D printer, and (c) the manufacturing process.

The experimental set up (schematic and actual photograph) is illustrated in Figure 2, where the PLA beam is attached to the end of a $96 \mathrm{~cm}$ long vibrating aluminum bar to be excited by it. As the 
plastic materials possess low stiffness, it is hard to have them self-excited by an instantaneous, short pulse, as done using metallic beams in past experiments. Thus, the aluminum bar served as the external stimulus, exciting the beam at fixed frequencies. The vibrations on the aluminum bar were imposed using a mechanical vibrator, which was driven by a 10 Volt waveform generator (AGILENT 33210A) with simple sinusoidal output. A press (CARVER 10 ton) was used for holding one side of the aluminum bar fixed (at lower pressure, the results are affected as the anchoring is loose), and two coils were wrapped around the beam sample, one serving as a detection coil on which an EMF voltage was generated due to the varying magnetic flux produced by the magnetoelastic material, and the other as a bias coil providing a constant DC magnetic field to magnetize the Metglas ribbons. Alternatively, for more practical applications, a permanent magnetic bar can be used in close proximity to the magnetoelastic sensors as the DC biasing source, as it is done in the magnetoelastic anti-theft tags. The EMF voltage was recorded using a KEITHLEY-2000 voltmeter, and it was found to be of the order of a few millivolts. The bias coil was driven by a constant current source (TTi QL355P) set at $I=0.2 \mathrm{~A}$.
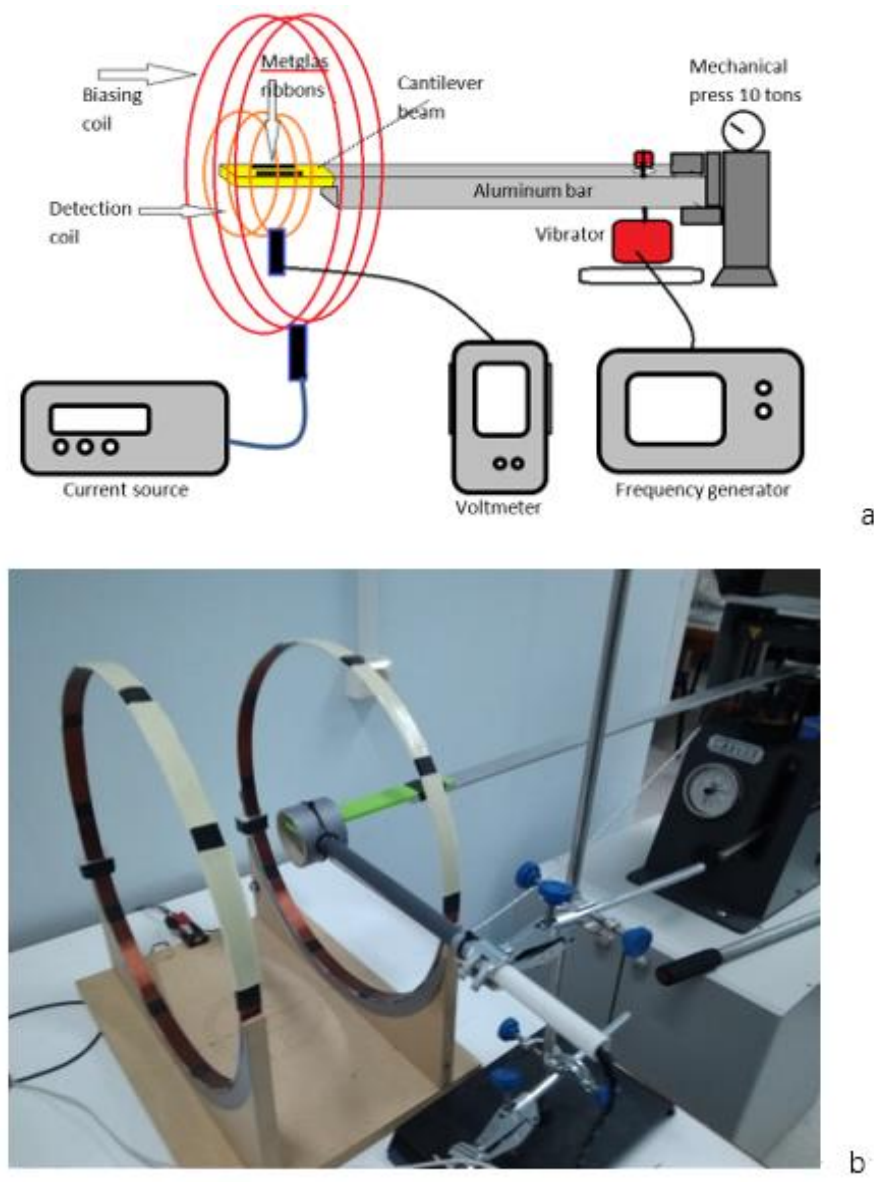

Figure 2 Experimental setup (a) schematic and (b) actual photo.

\section{Results}

\subsection{Natural Frequencies}

The purpose of the current work is to examine the hypothesis, in a proof-of-concept manner, that embedded metallic-glass ribbons inside a beam can act as sensors and deliver useful 
information such as the spectrum of the natural bending frequencies. The study also inspects the dependence of the depth of the ribbons inside the beam with respect to the signal intensity, along with its effects on the emitted spectrum.

In order to achieve this goal, the frequency generator shown in Figure 2 was operated manually between $170 \mathrm{~Hz}$ and $1100 \mathrm{~Hz}$ with steps of $10-15 \mathrm{~Hz}$ when the signal was low. Further, it was operated with shorter steps down to $1 \mathrm{~Hz}$ when the signal was getting stronger, indicating the presence of a peak. The corresponding measurements are represented in Figure 3 where the sensing ribbons are placed at four different depths $d$, starting with $d=0$ at the beam's external top surface, all the way down to the beam's mid-plane at values of $d=1,2$, and $3 \mathrm{~mm}$. As the beam's thickness is $6 \mathrm{~mm}$ and it is symmetric around its mid-plane, there is no need to approach the larger depths below this plane as the results are similar to the ones above the plane.

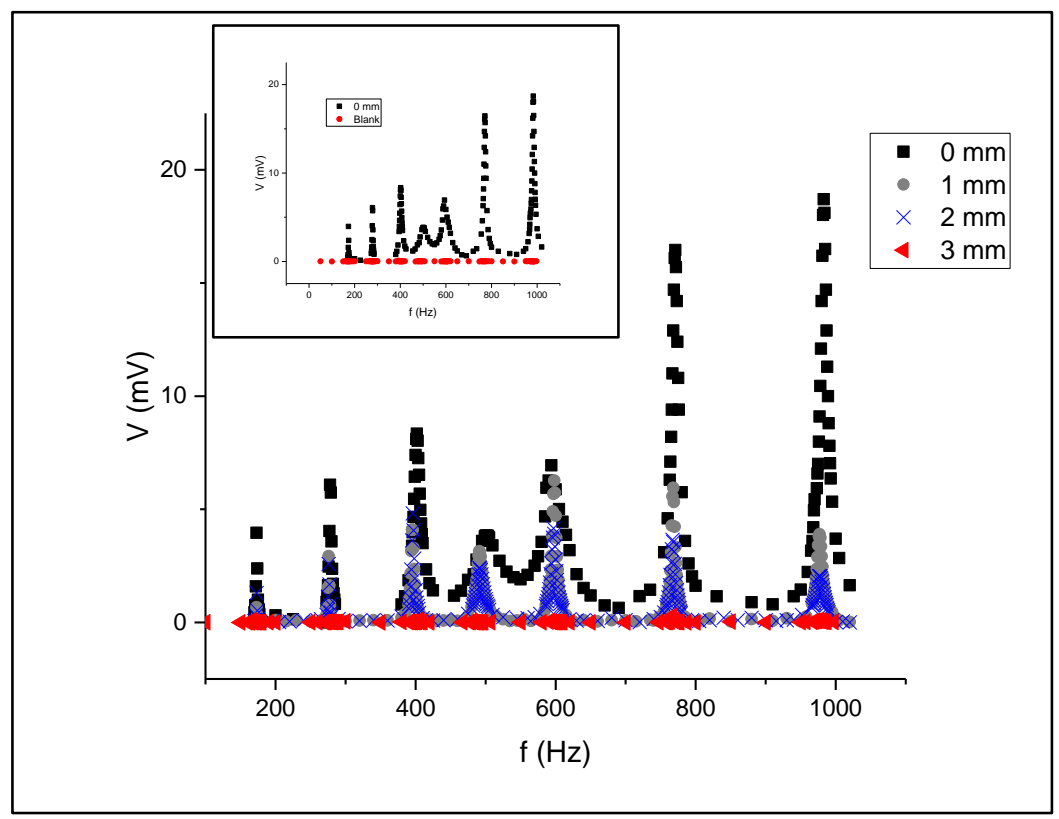

Figure 3 Frequency scan of bending vibrations of a plastic beam, with the sensing elements embedded in different depths of 0 (beam top surface), 1, 2, and $3 \mathrm{~mm}$. The inset compares the signal from the $0 \mathrm{~mm}$ beam (black points) with the blank beam (no sensor, red points).

It is obvious that the measured EMF voltage exhibits sharp maxima at certain frequencies, which possibly correspond to the harmonics of bending modes (this will be described in detail in the very next subsection). In order to ensure that the measured signal was generated by the sensing ribbons and not from some other external source of radiation, a control experiment was performed under the same conditions but with a beam without having any sensor mounted on it (blank beam), and the measured voltage was both insignificant down to the sub-millivolt range and showed no peaks (represented in the inset of Figure 3 together with the $0 \mathrm{~mm}$ data for comparison). Thus, it was concluded that the results of the experiments were solely due to the presence of the sensors. The peak frequency values shown in Figure 3 are mentioned in Table 3 (the results for $3 \mathrm{~mm}$ are not mentioned as the peaks are very low and thus possess large errors). It is clear that the mentioned technique is capable of detecting seven harmonics, and remarkably, these frequencies remain the same, within a marginal relative error of $1 \%$, for all depths. The error percentage is calculated as 
100 (MAX-MIN)/AVG/2, which indicates that it is equal to the difference of the maximum to the minimum of the three values in the same row (corresponding to the different depths) divided by 2 and by their average value. This relative error is small because the ribbon's thickness of $\mathbf{3 0}$ microns is negligible with respect to the beam's thickness of $6 \mathrm{~mm}$. It thus does not disturb the mechanical structure by introducing new boundary conditions (this point will be further elaborated and supported experimentally as well as theoretically in the next subsection).

Table 3 The peak frequency values in $\mathrm{Hz}$ of the spectra of Figure 1.

\begin{tabular}{llllll}
\hline $\begin{array}{l}\text { Depth } \\
(\mathrm{mm})\end{array}$ & 0 & 1 & 2 & Average & $\begin{array}{l}\% \\
\text { error }\end{array}$ \\
\hline Peak No & $f(\mathrm{~Hz})$ & $f(\mathrm{~Hz})$ & $f(\mathrm{~Hz})$ & $f(\mathrm{~Hz})$ & \\
1 & 173 & 173 & 173 & 173.0 & 0.0 \\
2 & 278 & 276 & 276 & 276.7 & 0.4 \\
3 & 402 & 396 & 396 & 398.0 & 0.8 \\
4 & 503 & 491 & 491 & 495.0 & 1.2 \\
5 & 594 & 598 & 597 & 596.3 & 0.3 \\
6 & 771 & 768 & 767 & 768.7 & 0.3 \\
7 & 983 & 977 & 977 & 979.0 & 0.3 \\
\hline
\end{tabular}

As derived from Figure 4, the detected amplitude of the different peaks almost halves going from the beam's surface $d=0$ to the first depth $d=1 \mathrm{~mm}$, and continues to drop to almost zero value at the midplane with an increase in the depth. Hence, at relatively shallow depths, the embedded ribbons can be efficiently used to derive the beam's harmonics. The above behaviors can be explained as follows:

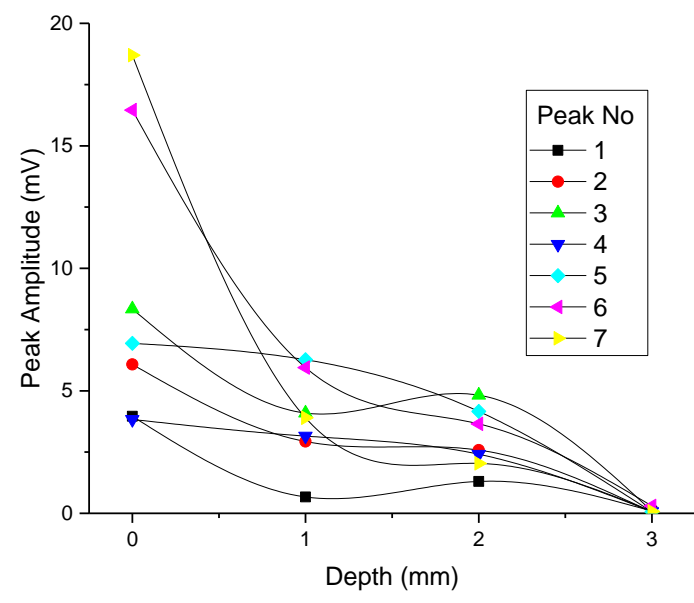

Figure 4 Peak Amplitudes of the seven peaks measured in Figure 3 as a function of the depth of the sensing ribbons. The numbers in the legend correspond to peak numbers and not the harmonics.

The sensor at $0 \mathrm{~mm}$ has a free surface and can oscillate unaffectedly. But the embedded sensors are surrounded by plastic material and are trapped. This imposes some damping to the PLA 
oscillation, justifying the small amplitudes in the spectra. Moreover, as the $d$ increases, the sensor approaches the central symmetry plain of the rod, which is considered as a strain-free plain in the classical theory of bending, which suggests less excitation for the sensors. Hence the drop of the signal with an increase in $d$ is expected from the stress arguments for a state of pure bending. Nevertheless, the signal is still strong at $1 \mathrm{~mm}$ and $2 \mathrm{~mm}$ to be used to extract the modes spectra, as demonstrated in Figure 3.

Concerning the peak amplitude of different harmonics in Figure 4, it seems that the higher the peak number, the higher the detection signal is, with the exception of the fourth peak number. At first, this observation seems to be in disagreement with an earlier study [28] where the reduction of the signal intensity of a similar magnetoelastic sensor was reported at higher resonance modes. However, there are two main differences between this work and the presented measurements: (a) the mentioned experiment is in a state of forced excitation by the external vibrator, which is set to a constant amplitude, which suggests that its energy is proportional to the frequency. This indicates that the higher harmonics receive more energy. In an earlier study [28], the sensor is first excited and then left to dissipate naturally in a "bell-ring" fashion. In such a case, the base harmonic is the stronger one. Quoting from the paper, "to monitor the 2nd and 3rd modes, higher DC biasing fields were used to improve signal quality", which signifies higher excitation energy, something that our vibrator does naturally in the constant amplitude mode. (b) Moreover, in an earlier study [28], the sensor is forced to vibrate longitudinally while our experiment is in the plain bending vibration mode. In this mode, it was observed in the previous work with single aluminum bars as cantilevers $[25,26]$ that the first $n=1$ and $n=2$ peaks were very weak compared to the higher peaks.

\subsection{Proof of Minimal Sensor Disturbance}

In this subsection, it will be proved experimentally as well as theoretically that the attachment of the thin Metglas ribbons is just a minimal disturbance to the beam structure, with respect to its mechanical properties. Additionally, the beam Elastic modulus will be extracted from the experimental data. In order to accomplish this, the experiment shown in Figure 5a was performed using a PLA cantilever beam similar to the one in the experiment of Figure 2 but with slightly different dimensions $L=11.6 \mathrm{~cm}$ (free length), $t=2.75 \mathrm{~mm}$, and $w=3 \mathrm{~cm}$. The beam was fixed as a cantilever with the help of a vial, and a light jar was suspended from the free end. A micrometer was attached at the free end to measure the vertical deflection $\delta$ at this point. Different amounts of water of known mass $\mathrm{m}$ were added to the jar, and the deflection was recorded. Figure $5 \mathrm{~b}$ represents the data plot of $\delta$ versus $m$ for the two cases of a beam with/without the Metglas ribbons attached to it. The Young modulus $E$ of the beam can be extracted from this dataset with the help of the known stiffness formula for cantilever beams:

$$
\delta=\frac{L^{3}}{3 E I} F
$$

Here $F$ is the applied vertical load at the free end, $L$ represents the free length, and $I$ signifies the second moment of area. For a beam with a rectangular cross-section of width $b$ and thickness $t, I=$ $b t^{3} / 12$ can be derived, and for the force of gravity, $F=m g$ is used. When these results are substituted into eq. 3 , they lead to the following result for the slope of the $\delta$ versus $m$ line: 


$$
\frac{\Delta \delta}{\Delta m}=\frac{4 g L^{3}}{E b t^{3}}
$$

The slope is hence inversely proportional to $\mathrm{E}$.
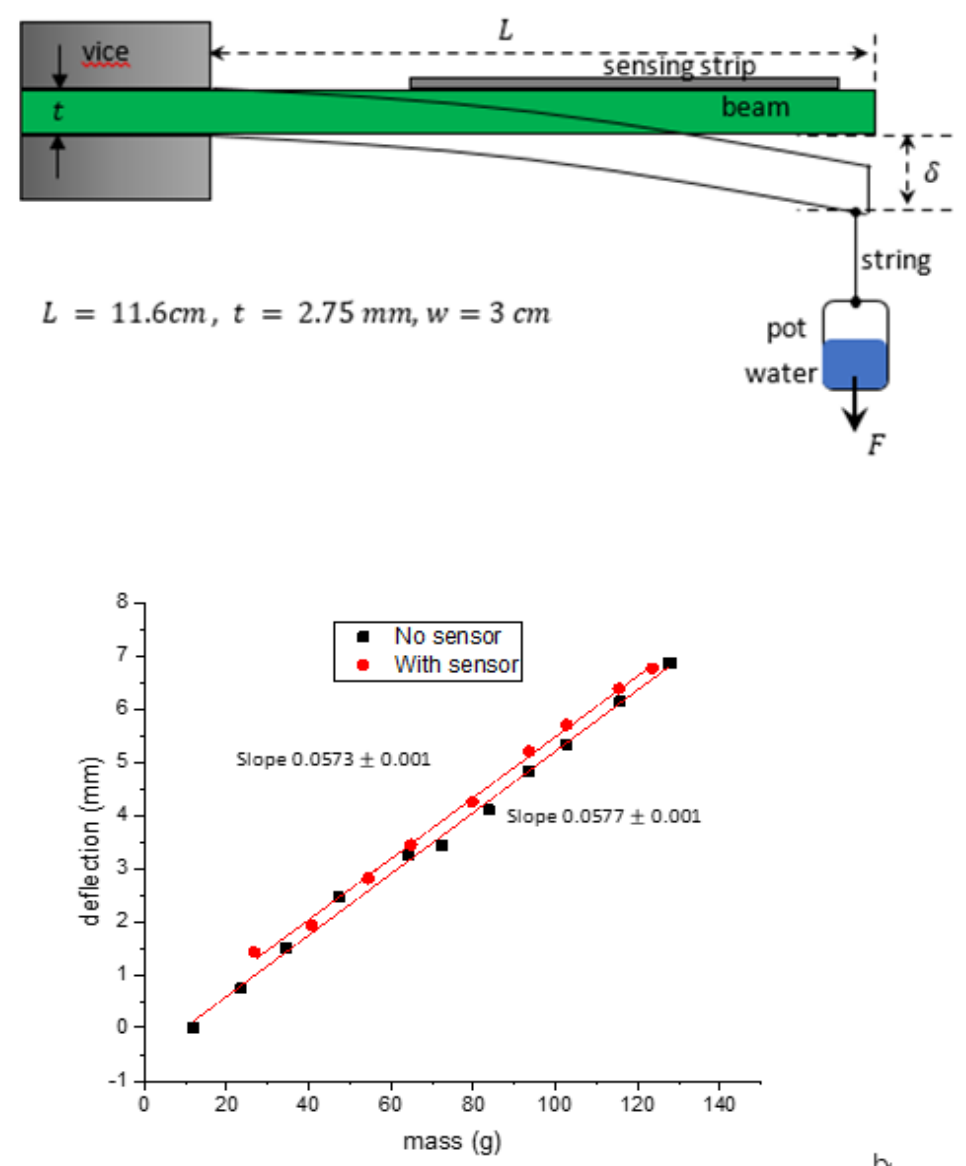

Figure 5 (a) Schematic of the experimental setup for the measurement of the elastic modulus $E$ and $(b)$ the data taken with this setup.

As demonstrated in Figure 5b, two sets of experiments were performed, one with and one without Metglas strips attached on the top of the beam. The technique of least square fit was used to compute the two slopes with values $0.0577 \pm 0.001 \mathrm{~mm} / \mathrm{g}$ and $0.0573 \pm 0.001 \mathrm{~mm} / \mathrm{g}$ for the cases of no sensor and sensor, respectively. Since the two slopes are equal within the experimental error, it can be concluded that the sensor attachment on the beam does not lead to a measurable disturbance on the mechanical properties of the beam.

From eq. (4) and the calculated slope of $0.0577 \pm 0.001 \mathrm{~mm} / \mathrm{g}$ for the "no sensor" case, the value of $E=1.70 \mathrm{GPa}$ was derived for the PLA beam. This value is not necessarily the value of the solid PLA material as the 3D printer creates solids using infill patterns with lower density than the actual solid material. The bar in Figure 2 possesses a mass of $25.92 \mathrm{~g}$ leading to a density of $0.72 \mathrm{~g} / \mathrm{cm}^{3}$. To have an estimate, a density of $1.25 \mathrm{~g} / \mathrm{cm}^{3}$ and a value of $E=3.5 \mathrm{GPa}$ (Table 1 therein) were found in the literature for PLA [29], which is almost twice as large as our values.

It can also be proved with simple theoretical arguments that the attachment of the thin ribbons has a minimal or no effect on the mechanical properties of the attached beam. A similar conclusion 
can be derived from laminar composite materials where it is well known that the upper limit of the elastic modulus of the composite is given by $E=V_{1} E_{1}+V_{2} E_{2}$, where $V_{1}$ and $V_{2}$ are the volume fractions of the two phases, and $E_{1}$ and $E_{2}$ are their corresponding moduli. For $200 \mathrm{~mm} \times 30 \mathrm{~mm} \times$ $6 \mathrm{~mm}$ PLA sample and $100 \mathrm{~mm} \times 1 / 4 \mathrm{in} \times 30 \mu \mathrm{m}$ Metglas ribbons, the volume fractions are computed to be 0.9994 and 0.0006 , respectively. Using the aforementioned Elastic modulus for PLA and from Table 2 for Metglas, a "composite" Elastic modulus can be received, which differs only $1 \%$ from the PLA modulus, which is about the difference observed in the slopes of Figure $5 \mathrm{~b}$ and is rather insignificant for practical purposes.

\subsection{Comparison with the Theory}

In Table 4, the average measured frequencies of Table 3 together with the $f_{n} / x_{n}{ }^{2}$ ratio are specified, where $x_{n}$ are the theoretical values of Table 1 . The best fit (roughly constant ratio) is identified by selecting the modes $n \geq 5$. It can be observed that with this choice, the $f_{n} / x_{n}{ }^{2}$ ratio is roughly constant with a value of $0.89 \pm 0.06$, from which it can be argued that the measured peaks correspond indeed to the bending modes $n=5,6, \ldots 11$. It is not clear why the $n \leq 4$ modes are missing; perhaps they are present but with lesser measured signal strength compared to the noise and thus more accurate experiments are required to reveal them. As it was mentioned above, in the previous work $[25,26]$, the $n=1$ and $n=2$ peaks were very weak, and perhaps the additional damping introduced by the plastic beam here causes two more peaks to disappear (to avoid confusion, it must be recorded that in Figure 4 the mode number $n$ corresponds to "peak number" +4).

Table 4 Comparison of the peak spectra of Figure 1 to theoretical values and the expected values for Aluminum and PLA bar

\begin{tabular}{lllllll}
\hline & Experiment & Mode & Ratio & Aluminum & Dev. Al & PLA \\
\hline Peak No & Avg $f_{n}(\mathrm{~Hz})$ & $n$ & $f_{n} / x_{n}{ }^{2}$ & $f_{n}(\mathrm{~Hz})$ & $\%$ & $f_{n}(\mathrm{~Hz})$ \\
1 & 173.0 & 5 & 0.87 & 252 & 46 & 2117 \\
2 & 276.7 & 6 & 0.93 & 376 & 36 & 3162 \\
3 & 398.0 & 7 & 0.95 & 525 & 32 & 4416 \\
4 & 495.0 & 8 & 0.89 & 699 & 41 & 5878 \\
5 & 596.3 & 9 & 0.84 & 898 & 51 & 7549 \\
6 & 768.7 & 10 & 0.86 & 1122 & 46 & 9429 \\
7 & 979.0 & 11 & 0.90 & 1371 & 40 & 11525 \\
\hline
\end{tabular}

Also, from Table 4, it can be analyzed that the corresponding values calculated from eq. 2 are the bending mode frequencies for the plain aluminum bar that was used in the discussed experiment, using its dimensions and standard Aluminum values of $E=69 \mathrm{GPa}$ and density of $2.7 \mathrm{~g} / \mathrm{cm}^{3}$. The column next to the Aluminum frequencies contains the percentage deviation of these frequencies to the measured frequencies, and it can be recorded that this deviation is within the range of $30 \%$ $50 \%$. Perhaps the plastic PLA beam attached on the end of the aluminum beam in Figure $2 \mathrm{~b}$ acts as damping to the Al beam with a damping factor within this range. For comparison, the corresponding peaks of the PLA bar are also mentioned in the last column of Table 4 above, using the density and 
E values found in the previous subsection. Evidently, these values are an order of magnitude higher than the measured mode frequencies, and hence the plastic beam does not come to bending resonance, but it just adds damping to the aluminum bar.

\section{Conclusions}

In the current work, magnetoelastic ribbons of 30-micron thinness made of metallic glass are embedded inside $6 \mathrm{~mm}$ thick PLA plastic cantilever beams made by 3-D printing to test whether they can act as vibration sensors from within the material by detecting the beam's harmonics during bending excitation. This is feasible as the ribbons exhibit magnetoelastic nature and thus changes in their mechanical state, inducing a corresponding change in their magnetic state. The measured EMF voltage picked by an external coil reports sharp maxima at certain frequencies, which probably correspond to the harmonics of bending vibrations of the combined system of the PLA beam and its stimulus aluminum beam. The ribbons are placed at four different depths in the PLA beam, starting with zero depth at the beam's external surface all the way inside the beam's mid-plane. This technique is capable of identifying seven harmonics. These frequencies remain remarkably the same within a marginal error of $1 \%$ for all the depths. This is because the ribbon's thickness of 30 microns is negligible with respect to the beam's thickness of $6 \mathrm{~mm}$, and thus it does not disturb the mechanical structure by introducing new boundary conditions. The detected amplitude of the modes reduces to almost half going from the beam's surface to the first depth of $1 \mathrm{~mm}$, and it continues to drop as the depth increases, all the way down to the beam's mid-plane, but it is still strong enough to provide information about the beam harmonics. This means that the embedded ribbons can be successfully used to detect the mechanical health of a structure, as the set of the harmonics is a unique signature of the structure's state, provided the ribbons are not placed too deep inside the structure. The merits of placing the sensing ribbons inside the material instead of placing it externally are as follows: (a) the information can be related to the internal state of the material, like internal cracks, which are difficult to be identified externally, (b) the protection of the ribbons from harsh environments, and (c) the creation of a composite material, which combines the properties of the host with the superb mechanical properties of the metallic glasses. A proof-ofconcept study is presented here to prove that this kind of sensing is possible using the sensors embedded in the host material while transmitting mechanical state information in a contactless fashion.

\section{Author Contributions}

D.K. and G.S conceived the idea, G.S. designed the experiment, G.S and C.I.T. performed the experiment, D.K. and G.S. analyzed the data and finally D.K. wrote the paper.

\section{Competing Interests}

The authors have declared that no competing interests exist.

\section{References}

1. Heise RE. Low-cycle fatigue-crack indications by strain gages operating in elastic strain fields. Exp Mech. 1965; 5: 19A-24A. 
2. Grosse CU. Acoustic emission localization methods for large structures based on beam forming and array techniques. Nantes: Proceedings of Non-Destructive Testing in Civil Engineering, Nantes (NDTCE'09); 2009.

3. Wang KC, Li Q. Applicability of microelectronic and mechanical systems (MEMS) for transportation infrastructure management. Fayetteville: Final Report for Project MBTC-2056, Department of Civil Engineering; 2008.

4. Pozzi M, Zonta D, Trapani D, Athanasopoulos N, Amditis AJ, Bimpas M, et al. MEMS-based sensors for post-earthquake damage assessment. J Phys Conf Ser. 2011; 305: 012100.

5. Yao Y, Tung ST, Glisic B. Crack detection and characterization techniques-An overview. Struct Control Health Monit. 2014; 21: 1387-1413.

6. Sundaresan MJ, Ghoshal A, Schulz MJ. A continuous sensor for damage detection in bars. Smart Mater Struct. 2002; 11: 475.

7. Lee JW, Kirikera GR, Kang I, Schulz MJ, Shanov VN. Structural health monitoring using continuous sensors and neural network analysis. Smart Mater Struct. 2006; 15: 1266.

8. Bae DY, Lee JR, A health management technology for multisite cracks in an in-service aircraft fuselage based on multi-time-frame laser ultrasonic energy mapping and serially connected PZTs. Aerosp Sci Technol. 2016; 54: 114-121.

9. Okafor AC, Dutta A. Structural damage detection in beams by wavelet transforms. Smart Mater Struct. 2000; 9: 906.

10. Jassim ZA, Ali NN, Mustapha F, Jalil NA. A review on the vibration analysis for a damage occurrence of a cantilever beam. Eng Fail Anal. 2013; 31: 442-461.

11. Zhou Z. Vibration based damage detection of simple bridge superstructures. Saskatoon: University of Saskatchewan; 2006.

12. Maeck J. Damage assessment of civil engineering structures by vibration monitoring. PhD thesis. Universiteit Leuven; 2003.

13. Pandey AK, Biswas M, Samman MM. Damage detection from changes in curvature mode shapes. J Sound Vib. 1991; 145: 321-332.

14. Adams RD, Walton D, Flitcroft JE, Short D. Vibration testing as a non-destructive test tool for composite materials. Philadelphia: American Society for Testing Materials. 1975; 580: 159-175.

15. Loland O, Dodds CJ. Experiences in developing and operating integrity monitoring system in North Sea. Houston: Presented at the Eighth Annual Offshore Technology Conference; 1976.

16. Vandiver JK. Detection of structural failure on fixed platforms by measurements of dynamic response. Offshore Technology Conference; 1975.

17. Yuen MM. A numerical study of the eigenparameters of a damaged cantilever. J Sound Vib. 1985; 103: 301-310.

18. Dimarogonas AD. Vibration Engineering. Minnesota: West Publishers; 1976.

19. Chondros T. Dynamic response of cracked beams. Greece: University of Patras; 1977.

20. Chondros TG, Dimarogonas AD. Identification of cracks in welded joints of complex structures. J Sound Vib. 1980; 69: 531-538.

21. Chondros TG, Dimarogonas AD. Identification of cracks in circular plates welded at the contour. American Society of Mechanical Engineers Design Engineering Technical Conference; 1979.

22. Cawley P, Adams RD. Defect location in structures by a vibration technique. American Society of Mechanical Engineers Design Engineering Technical Conference; 1979. 
23. Anifantis N, Aspragathos N, Dimarogonas AD. Diagnosis of cracks on concrete frames due to earthquakes by vibration response analysis. Moscow: Third International Symposium of the International Measurements Federation (IMEKO); 1983.

24. Inagaki T, Kanki H, Shiraki K. Transverse vibrations of a general cracked-rotor bearing system. J Mech Design. 1981; 345-355.

25. Samourgkanidis G, Kouzoudis D. Experimental detection by magnetoelastic sensors and computational analysis with finite elements, of the bending modes of a cantilever beam with minor damage. Sens Actuator A Phys. 2018; 276: 155-164.

26. Samourgkanidis G, Kouzoudis D. A pattern matching identification method of cracks on cantilever beams through their bending modes measured by magnetoelastic sensors. Theor Appl Fract Mech. 2019; 103: 102266.

27. Chiriac $\mathrm{H}$, Pletea M, Hristoforou E. Fe-based amorphous thin film as a magnetoelastic sensor material. Sens Actuator A Phys. 2000; 81: 166-169.

28. DeRouin A, Ong KG. Multi-parameter sensing with a single magnetoelastic sensor by applying loads on the null locations of multiple resonant modes. Smart Mater Struct. 2016; 25: 035044

29. Farah S, Anderson DG, Langer R. Physical and mechanical properties of PLA, and their function in widespread applications-A comprehensive review. Adv Drug Deliv Rev. 2016; 107: 367-392.

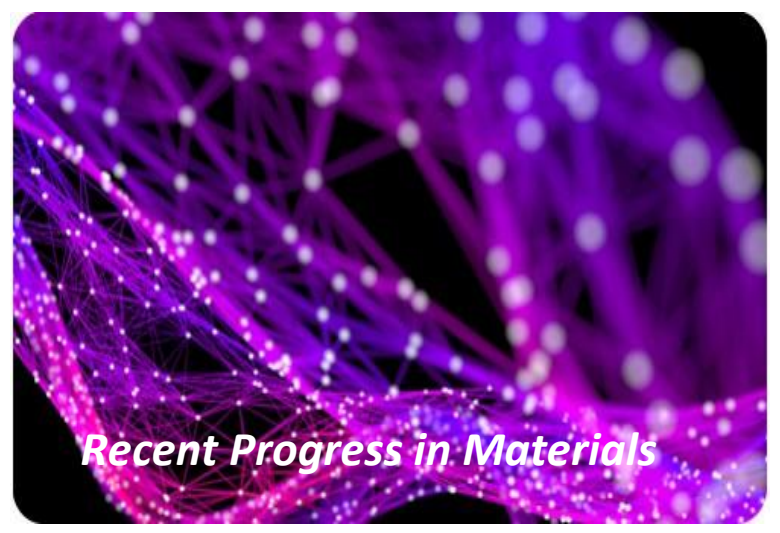

Enjoy Recent Progress in Materials by:

1. Submitting a manuscript

2. Joining in volunteer reviewer bank

3. Joining Editorial Board

4. Guest editing a special issue

For more details, please visit: http://www.lidsen.com/journals/rpm 